\title{
Numerical Experiments for Nuclear Flashes toward Superbursts in an Accreting Neutron Star
}

\author{
Masa-aki Hashimoto, ${ }^{1}$ Reiko Kuromizu, ${ }^{1}$ Masaomi Ono, ${ }^{1}$ \\ Tsuneo Noda, ${ }^{2}$ and Masayuki Y. Fujimoto ${ }^{3}$ \\ ${ }^{1}$ Department of Physics, Kyushu University, Fukuoka 810-8560, Japan \\ ${ }^{2}$ Kurume Institute of Technology, Fukuoka 830-0052, Japan \\ ${ }^{3}$ Department of Physics, Hokkaido University, Sapporo 060-8810, Japan \\ Correspondence should be addressed to Masa-aki Hashimoto; hashimoto@phys.kyushu-u.ac.jp
}

Received 10 March 2014; Revised 20 June 2014; Accepted 20 June 2014; Published 23 July 2014

Academic Editor: Luciano Nicastro

Copyright (C) 2014 Masa-aki Hashimoto et al. This is an open access article distributed under the Creative Commons Attribution License, which permits unrestricted use, distribution, and reproduction in any medium, provided the original work is properly cited.

\begin{abstract}
We show that the superburst would be originated from thermonuclear burning ignited by accumulated fuels in the deep layers compared to normal X-ray bursts. Two cases are investigated for models related to superbursts by following thermal evolution of a realistic neutron star: helium flash and carbon flash accompanied with many normal bursts. For a helium flash, the burst shows the long duration when the accretion rate is low compared with the observation. The flash could become a superburst if the burning develops to the deflagration and/or detonation. For a carbon flash accompanied with many normal bursts, after successive 2786 normal bursts during $1.81 \times 10^{9} \mathrm{~s}$, the temperature reaches the deflagration temperature. This is due to the produced carbon which amount reaches to $\approx 0.1$ in the mass fraction. The flash will develop to dynamical phenomena of the deflagration and/or detonation, which may lead to a superburst.
\end{abstract}

\section{Introduction}

Type I X-ray bursts have been identified to the thermonuclear explosions on the surface region of accreting neutron (compact) stars. As a consequence, the phenomenon has been studied from both nuclear reactions and nuclear structure inside the compact stars. However, there still remain many uncertainties concerning the elementary processes associated with the bursts [1-3]. Superbursts have been detected from $13 \mathrm{X}$-ray bursters by BeppoSAX and RXTE (see, e.g., Table 2 in [4]). In particular, $4 \mathrm{U}$ 1636-536 exhibited four superbursts, where the shortest recurrence time is 1.5 years (http://www.astronomerstelegram.org/?read=2140) $[5$, 6]. Clearly, the light curve consists of a fast rise and slower power law-like decay $[2,7]$. The spectrum hardens during the rise phase to the maximum in luminosity, whereas it softens in the decay phase. This is also reflected in the spectral fits to the time-resolved preburst subtracted from Xray spectra. Each burst has energy of $10^{42}$ ergs and duration of a few hours. They are usually best described in terms of a black-body model. The effective temperature increases and decreases during the rise and decay phase, respectively. These superbursts are 1000 times luminous and 1000 times long in the duration compared with the normal bursts though the spectral evolution is similar.

Even now, quantitative explanation and/or numerical simulation of superbursts using the stellar evolution code are limited. For example, Keek and Heger [8] do not self-consistently produce the carbon from hydrogen/helium burning in their calculation but instead accrete the carbon directly onto the neutron star, bypassing the hydrogen/helium burning stages. The superbursts last too long and their energy release is too much to be explained in terms of unstable burning of hydrogen/helium so far considered [9]. Moreover, regular normal X-ray bursts are observed before the occurrence of the superburst [10] that includes the precursor burst [11]. The long rise and decay times of superbursts are consistent with the model of unstable burning 
in the deep layer below the hydrogen/helium burning region. Therefore, it has been suggested that unstable burning of carbon is the origin of the superbursts $[9,12]$.

If the accreted material onto the neutron star is pure helium, carbon can be produced when helium is burned stably [9]. Unstable helium burning often involves alpha captures on carbon and carbon does not remain much. This would apply to the helium accretor 4 U 1820-30 that shows long periods of high X-ray intensity during which no burst occurs, which is consistent with a period of stable helium burning. Note that unstable carbon burning only reproduces the observed feature in superbursts when we take into account neutrino losses and significant heat flux transported from deeper into the accreting layer of the neutron star [9]. Cumming et al. [2] also show that the observed superburst energy is around $10^{42}$ ergs and more or less independent of ignition depth because of neutrino emission of the excess energy. While Cumming [13] expected recurrence times of the order of 1-2 years, Strohmayer and Brown [9] obtained a recurrence time of about 10 years.

If the accreted material onto the neutron star is a mixture of hydrogen and helium, either unstable or stable burning of hydrogen/helium can produce carbon. While the amount obtained by numerical calculations has been only limited $[14,15]$ after bursts, carbon is much more readily produced in stable burning [1]. In the observed sources of superbursts, normal XRBs have been observed with a mean rate of about 3 times per day during the period of the observation [16, 17]. This indicates that at least some amounts of the accreted material have been burned stably before a superburst. Furthermore, the superburst from $4 \mathrm{U}$ 1254-69 indicates that much of the accreted fuel burns stably [18]. Detection of superbursts at near Eddington accretion rate would reveal the relation between the recurrence time and remained nuclear fuels, which is studied by using the $\alpha$ parameter [19].

Cumming and Bildsten [12] suggested that a small amount of carbon $\left(X\left({ }^{12} \mathrm{C}\right) \approx 0.05-0.1\right)$ could be enough to trigger a thermonuclear runaway with energy comparable to the superburst if carbon resides in a bath of heavy elements. These heavy elements are the products of unstable burning through the rp-process during the mixed hydrogen/helium burning of XRBs [14, 20]. In this case, the superburst recurrence time would depend on accretion rates, being in the order of a few decades, a year to a decade, or a week to a month, according to the accretion rate which is about 0.1 , 0.3 , or 1 times the Eddington accretion rate. On the other hand, it is proposed from the analysis of the photospheric radius expansion that an accretion rate may change during the burst by some factors [21]. Changes in accretion rates are also considered from recent study of the outburst of a transient X-ray binary [22]. Therefore, it is reasonable to include accretion rate variations in numerical calculations because the observations indicate that the luminosity of accreting neutron stars is variable. It has been suggested that the high temperature reached during a superburst induced the photodisintegration [23]. As a consequence, they got energy generations comparable to those due to the carbon induced superburst.
Another scenario was proposed by Kuulkers et al. [17]. They suggested that hydrogen left after the burning in the hydrogen/helium layer is reignited by the electron capture that is followed by successive captures of neutrons by heavy nuclei occurred in the deeper layer. The recurrence time of superbursts is estimated to be less than one year [10, 17]. However, large amounts of hydrogen should be remained after bursts to explain the energy release in superbursts. Recent calculations have revealed that hydrogen is completely depleted after the hydrogen/helium burning $[14,15]$. On the other hand, an exotic process of the diquark pair formation was proposed to explain superbursts [24].

On the other hand, significant progress has been done so far concerning the construction of model to study the phenomena of neutron star; two dimensional hydrodynamical model calculations of X-ray bursts $[25,26]$, study of the propagation of deflagration wave of a rapidly rotating neutron star [27], magnetorotational study [28, 29], and rotating hot-spot model examination of rotating neutron star [30] concerning burst ocillations. Unfortunately, it would be insufficient to study X-ray bursts in details beyond spherically symmetric model. Since there is no model in the literature that self-consistently calculates the production of carbon in hydrogen/helium and its subsequent ignition as superburst, we perform evolutionary calculations adopting spherically symmetric models of accreting neutron stars with important physical processes included [31].

In Section 2 physical inputs and our evolutionary code are explained. A helium flush model is presented in Section 3 related to superbursts. In Section 4, we present a model of carbon flash accompanied with many normal bursts of $\mathrm{H} / \mathrm{He}$ combined burnings and show the possibility of a superburst. Concluding remarks are given in Section 5.

\section{Evolution Code of a Neutron Star}

The general relativistic evolutionary equations of spherical stars in hydrostatic equilibrium as formulated by Throne [32] are written as

$$
\begin{gathered}
\frac{\partial M_{t r}}{\partial r}=4 \pi r^{2} \rho_{t}, \\
\frac{\partial P}{\partial r}=-\frac{G M_{t r} \rho_{t}}{r^{2}}\left(1+\frac{P}{\rho_{t} c^{2}}\right)\left(1+\frac{4 \pi r^{3} P}{M_{t r} c^{2}}\right) \mathscr{V}^{2}, \\
\frac{\partial\left(L_{r} e^{2 \phi / c^{2}}\right)}{\partial M_{r}}=e^{2 \phi / c^{2}}\left(\varepsilon_{\mathrm{n}}-\varepsilon_{v}-e^{-\phi / c^{2}} T \frac{\partial s}{\partial t_{\infty}}\right), \\
\frac{\partial \ln T}{\partial \ln P}=\min \left(\nabla_{\mathrm{rad}}, \nabla_{\mathrm{ad}}\right), \\
e^{-\phi / c^{2}} \frac{\partial Y_{i}}{\partial t_{\infty}}=\alpha_{i}, \\
\frac{\partial M_{t r}}{\partial M_{r}}=\frac{\rho_{t}}{\rho} \mathscr{V}^{-1}, \\
\frac{\partial\left(M_{t r}+4 \pi r^{3} P / c^{2}\right)}{4 \pi r^{4} \rho_{t}}=\frac{\mathscr{V}^{2}}{\partial r}
\end{gathered}
$$


where

$$
\mathscr{V} \equiv\left(1-\frac{2 G M_{t r}}{c^{2} r}\right)^{-1 / 2}
$$

The basic quantities are defined as follows: $\rho$ : rest mass density, $T$ : temperature, $Y_{i}$ : abundance of the $i$ th particle, $M_{r}$ : proper mass inside the radius $r, t_{\infty}$ : Schwarzschild time coordinate (proper time at a distant observer), $M_{t r}$ : total mass inside the radius $r$, $\phi$ : gravitational potential, $L_{r}$ : local luminosity, $P$ : pressure, $\rho_{t}$ : total nongravitational mass-energy density in mass units, $s$ : specific entropy, $\varepsilon_{n}$ : heating rate by nuclear burning, $\varepsilon_{\gamma}$ : cooling rate by escaping neutrinos, $\alpha_{i}$ : nuclear reaction rate for the $i$ th particle, and $\nabla_{\text {rad }}\left(\nabla_{\mathrm{ad}}\right)$ : the radiative (adiabatic) temperature gradient. In the accretion layer, the Eulerian coordinate (the mass fraction coordinate with changing mass $\left.q\left[=M_{r} / M(t)\right]\right)$ is used, which is the most suitable method for computations of stellar structure when stellar mass $(M)$ varies [33]. In our calculation, the mixing length theory of convection with the mixing length equal to the pressure scale height has been used [34]. The radiative zero boundary conditions are imposed at the outer boundary. An outermost mesh point, which is regarded as the photosphere, is given at $q=1-4.1 \times 10^{-20}$ [35].

The above set of general relativistic equations for the evolution of spherically symmetric stars has been solved by using a Henyey-type numerical scheme of implicit method. We adopt the evolution code of a spherically symmetric neutron star $[35,36]$. The star is divided into 266 meshes of the Lagrange mass-coordinate. The gravitational mass and radius of the neutron star are initially set to be $1.3 M_{\odot}$ and $8.1 \mathrm{~km}$, respectively. Then we have $\log g_{s}=14.56$ for the gravitational acceleration at the surface of the neutron star $g_{s}$. The depth of the accretion layer is $\sim 10 \mathrm{~m}$ and consists of 170 meshes. In the region of the combined hydrogen/helium burning, the interval between meshes is typically $5-6 \mathrm{~cm}$. Physical inputs and an approximation network are almost the same as adopted by Fujimoto et al. [31] except for the changes of reaction rates [37] and alpha-network used in Section 3. It should be noted that our approximation network has been constructed based on the one-zone calculation with use of a large network for the bursts [38, 39]. Our network includes 16 nuclei: ${ }^{1} \mathrm{H},{ }^{4} \mathrm{He},{ }^{12} \mathrm{C},{ }^{14} \mathrm{O},{ }^{15} \mathrm{O},{ }^{16} \mathrm{O},{ }^{17} \mathrm{~F},{ }^{22} \mathrm{Mg},{ }^{30} \mathrm{~S},{ }^{56} \mathrm{Ni},{ }^{60} \mathrm{Ni}$, ${ }^{60} \mathrm{Zn},{ }^{64} \mathrm{Zn},{ }^{64} \mathrm{Ge},{ }^{68} \mathrm{Ge}$, and ${ }^{68} \mathrm{Se}$. This network can be used till the hydrogen decreases until around $10 \%$ in mass fractions [39].

Although input physics has been described in detail [35], we briefly write it again. This is because input physics is crucial to determine the neutron star properties and it is closely related to the study of elementary processes of high density matter. As for the equation of state of outer part of the neutron star $\left(\rho<5 \times 10^{7} \mathrm{~g} \mathrm{~cm}^{-3}\right)$, an ideal gas plus radiation is assumed with the electron degeneracy and the Coulomb liquid correction included [40]. For the inner part, the equation of state has been constructed by Richardson et al. [41] based on Canuto [42, 43]. Neutrino emissivities include bremsstrahlung of nucleons [44] and electron-ion [45], and electron-positron pair, photo, and plasmon processes [46].
Opacities for iron- and neutron-rich material include the radiative opacities for ${ }^{56} \mathrm{Fe}$ by Malone [47] and the thermal conductivities [48-50]. For the opacities of lighter elements the analytical approximations by Iben [51] are adopted to the radiative ones $[52,53]$, to the thermal conductivity for a nonrelativistic electron gas of [54], and to that for a relativistic one [55], respectively. Screening effects of nuclear reactions are taken from Dewitt et al. [56]. In the present paper, we include the crustal heating [57],

$$
Q_{i}=6.03 \times \dot{M}_{-10} q_{i} 10^{33} \mathrm{ergs} \mathrm{g}^{-1},
$$

where $i$ is the number of the reaction, $\dot{M}_{-10}$ is the mass accretion rate in units of $10^{-10} M_{\odot} \mathrm{yr}^{-1}$, and $q_{i}$ is the number of the effective heat energy per nucleon in $\mathrm{MeV}$ for the $i$ th reaction which is tabulated in their paper.

We assume that material is accreted with the same entropy at the stellar surface, neglecting the surface effects caused by the accretion flow. Within the framework of the spherical symmetry, the kinetic energy of the falling material has little influence on the structure in the layer as deep as the burning shell, since the radial motion will be dissipated in the surface layers [35].

Initial models have been constructed through the continuous accretion ( $\dot{M}=$ constant) without nuclear burning until the steady state is achieved, where the nonhomologous part of the gravitational energy release vanishes [35].

\section{Simple Features of Models Concerning Superbursts-Helium Flash Model}

Superbursts observed in $4 \mathrm{U} 1820-30$ are considered to be accreted by pure helium, while $4 \mathrm{U}$ 1636-536, $4 \mathrm{U}$ 1735-44, and $4 \mathrm{U} 1254-690$ are accreted by hydrogen/helium [58]. The difference of the accretion matter may affect the mechanism of superbursts. Therefore, we first examine rather simplified models of superbursts, which are triggered by a helium flash. We note that deep helium ignition towards superbursts was discussed by Kuulkers et al. [59] on the X-ray binary $4 \mathrm{U}$ 0614+091. For the carbon flash, we also investigate realistic situation accompanied with many normal bursts in Section 4 .

The superburst of $4 \mathrm{U} 1820-30$ was observed in the duration of $2.5 \mathrm{hr}$, where the burst energy is $1.4 \times 10^{42} \mathrm{ergs}$ [9], and the peak luminosity is $L_{\text {peak }} \simeq 3.4 \times 10^{38} \mathrm{ergs}^{-1}$ [17]. To follow a pure helium flash, we use an alpha-network consisted of alpha-nuclei up to ${ }^{56} \mathrm{Ni}$, where the nuclear reaction rates are taken from Angulo et al. [37]. This network includes not only $(\alpha, \gamma)$ reactions but also $(\alpha, p)$ reactions; the latter in competition with the former is assumed to follow $(p, \gamma)$ reactions instantaneously. From Figure 1, we can see that although $L_{\text {peak }}$ is consistent with the observation for a high accretion rate, $\dot{M}=3 \times 10^{-8}$, and intermediate rate, $3 \times$ $10^{-9} M_{\odot} \mathrm{yr}^{-1}$, the duration of the burst is too short to explain the burst energy. It should be noted that $L_{\text {peak }}$ attains the Eddington limit as described in Hanawa and Fujimoto [36]. On the other hand, for a low accretion rate, $\dot{M}=3 \times$ $10^{-10} M_{\odot} \mathrm{yr}^{-1}$, we have obtained the burst energy around $10^{42}$ ergs which lasts more than $3 \mathrm{hr}$ (see Figure 1). It is noted 


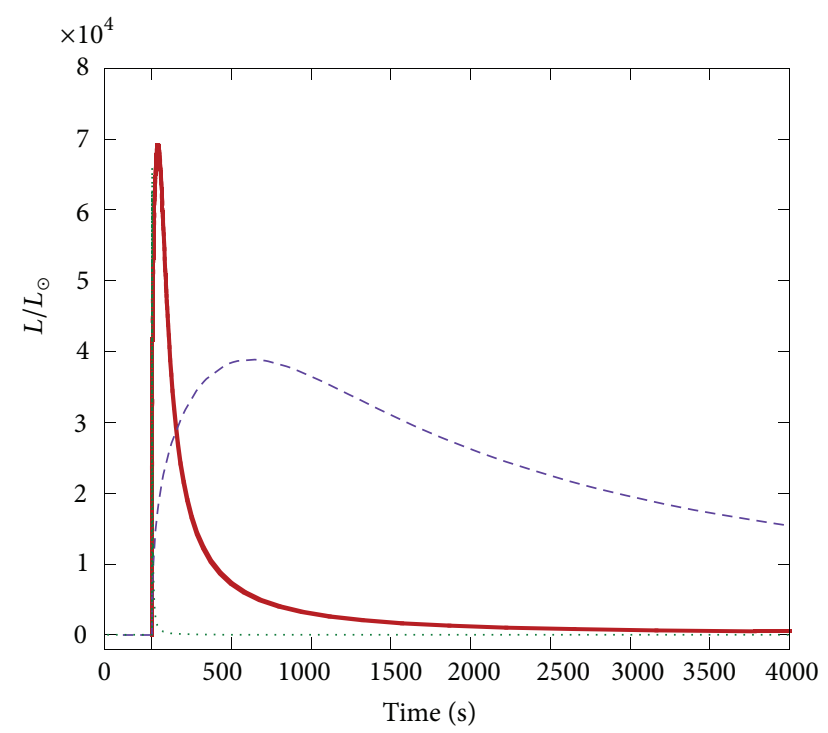

FIGURE 1: Light curves for three representative accretion rates, $\dot{M}=$ $3 \times 10^{-8}$ (dotted line), $3 \times 10^{-9}$ (solid line), and $3 \times 10^{-10} M_{\odot} \mathrm{yr}^{-1}$ (dashed line). Time is set to be zero at the beginning of each burst.

that the ignition pressures in units of dyn $\mathrm{cm}^{-2}$ for the above accretion rates are $10^{22.7}, 10^{24.1}$, and $10^{26.5}$, respectively. Since $\dot{M}$ of the superburst in $4 \mathrm{U} 1820-30$ is estimated to be several times $10^{-9} M_{\odot} \mathrm{yr}^{-1}$ [13], we recognize that simple models based on the single burst with an accretion rate assumed for the accreting neutron star are inconsistent with the observation (see Section 4). However, since the ultracompact source $4 \mathrm{U} 0614+91$ likely accretes helium at $10^{-10} M_{\odot} \mathrm{yr}^{-1}$ with a superburst [59], we could carefully study the helium flash.

Figure 2 shows the temperature distribution for $\dot{M}=3 \times$ $10^{-10} M_{\odot} \mathrm{yr}^{-1}$ against the density of the initial state (dotted line) and that of the stage at the maximum nuclear luminosity $L_{\mathrm{n}, \max }$ (solid line), where $L_{\mathrm{n}}=\int \varepsilon_{\mathrm{n}} d M_{r}$ denotes the nuclear luminosity. The dashed line indicates the ignition curve of the $3 \alpha$ reaction $\varepsilon_{3 \alpha}=\varepsilon_{\text {rad }}$ for the helium mass fraction $Y=0.1$, with the nuclear energy generation rate of $3 \alpha$ reaction $\varepsilon_{3 \alpha}$ and the radiative energy loss rate $\varepsilon_{\text {rad }}$ [14]. Although this criterion should be carefully reconsidered [60], we adopt the present one for simplicity. The dotted line is the deflagration temperature defined by equating the dynamical time scale and the nuclear heating time scale $\left(\tau_{\text {dyn }}=\tau_{\mathrm{n}}\right)$ with

$$
\tau_{\text {dyn }}=\frac{H_{p}}{c_{s}}, \quad \tau_{\mathrm{n}}=\frac{C_{p} T}{\varepsilon_{\mathrm{n}}},
$$

where $H_{p}(\equiv-d r / d \ln P)$ is the pressure scale height, $c_{s}$ is the sonic velocity, and $C_{p}$ is the specific heat under the constant pressure. It is remarkable that the temperature in the layers of $\log \rho=8.8$ exceeds $\log T=8.5$ for $L_{\mathrm{n} \text {, max }}$ : the flash may become the deflagration. It needs to perform a dynamical calculation to elucidate how the deflagration develops inside the accretion layers. Although we cannot represent the proper $L_{\text {peak }}$ consistent with the observation of $\dot{M}$, the helium flash

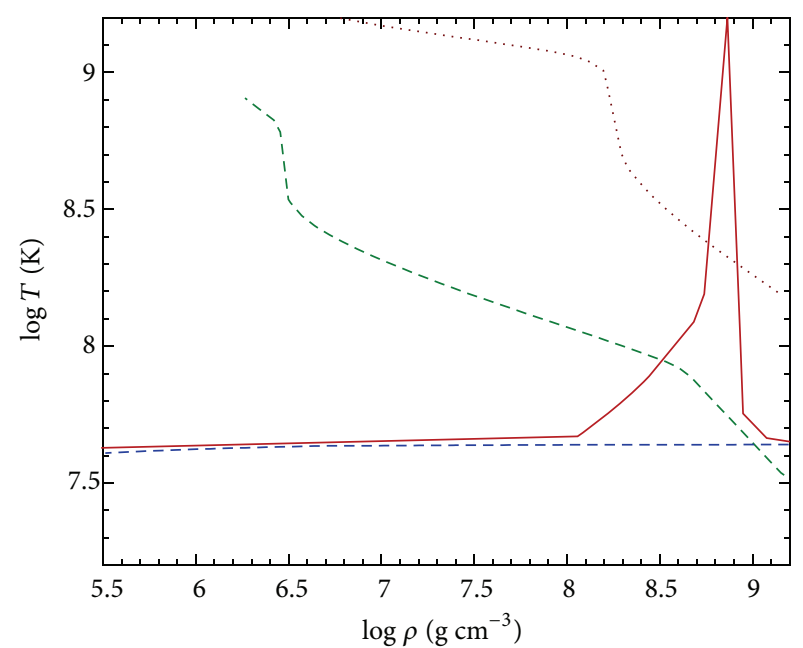

FIgURE 2: Temperature distributions for $\dot{M}=3 \times 10^{-10} M_{\odot} \mathrm{yr}^{-1}$ in the initial state (lower dotted line) and to the stage of the maximum $L_{\mathrm{n}}$ (solid line). The dashed line is the ignition curve $(Y=0.1)$ and the upper dotted line indicates that $\tau_{\mathrm{dyn}}=\tau_{3 \alpha}$.

in low accretion rate could become a possible site of the superburst.

\section{Model Accompanied with Normal Bursts of Combined Hydrogen and Helium Burnings}

In the previous section, we have shown that simple helium burst models cannot explain the observed superbursts. New model is needed to produce enough carbon for a superburst to occur. Therefore, we present a sequence of calculations until the amount of carbon increases enough. We first adopt an accretion rate of $5 \times 10^{-9} M_{\odot} \mathrm{yr}^{-1}$. And to acquire more carbon, we only change it to $1 \times 10^{-9} M_{\odot} \mathrm{yr}^{-1}$, which save the computational time. To increase the temperature in the distribution, we change the accretion rate to the first one. Furthermore, we also raise the crustal heating by a factor of ten to save the computational time.

4.1. Gross Features toward the Carbon Flash. Let us make an initial model to simulate a superburst with the accretion rate of $5 \times 10^{-9} M_{\odot} \mathrm{yr}^{-1}$. This accretion rate is considered to be in the reasonable range of $0.1 \leq \dot{M} / \dot{M}_{\text {Edd }} \leq 0.3$ with $\dot{M}_{\text {Edd }}=1.7 \times 10^{-8} M_{\odot} \mathrm{yr}^{-1}$ for the observed sources [61]. Note that superbursts at near-Eddington rate are suggested for GX $17+2$ [19]. With the nuclear burning suppressed, we construct an initial temperature distribution by continuous accretion with this specified $\dot{M}$. Mass fractions in the accretion matter are assumed to be $\mathrm{H}(73.0 \%),{ }^{4} \mathrm{He}(25.0 \%),{ }^{14} \mathrm{O}(0.7 \%)$, and ${ }^{15} \mathrm{O}(1.3 \%)$. The bottom of the accretion layer is set to be pure ${ }^{56} \mathrm{Ni}$, which is equivalent to ${ }^{56} \mathrm{Fe}$ in the present purpose. The steady state concerning the accretion rate is assumed; that is, $\dot{M}=\dot{M}_{56 \mathrm{Ni}}$; this means that accreted matter increases the mass of the layer of pure ${ }^{56} \mathrm{Ni}$; that is, accreting matter becomes promptly ${ }^{56} \mathrm{Ni}$ and omits nuclear burning processes. 


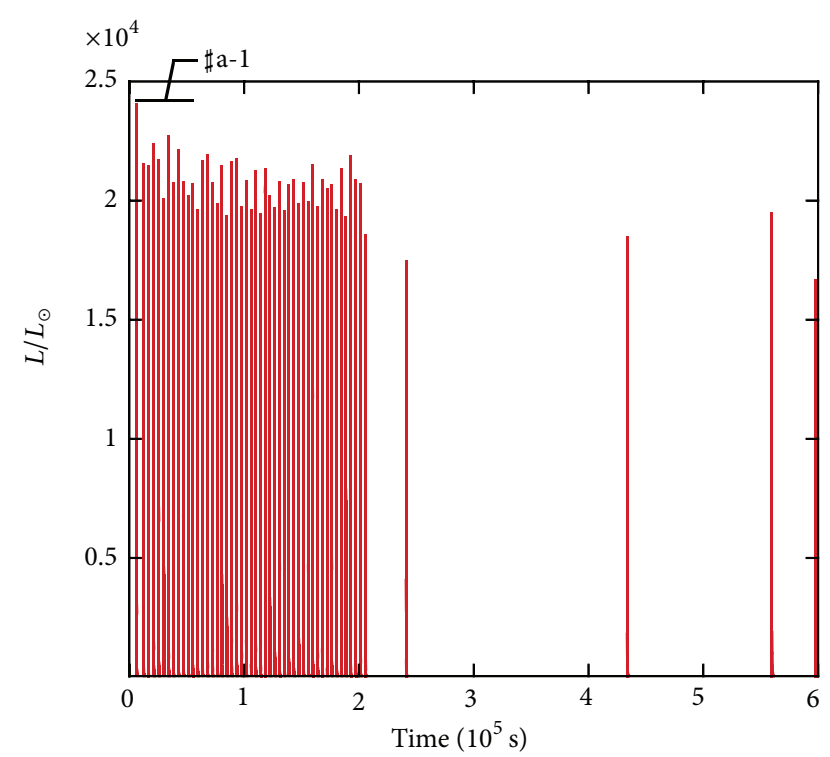

Figure 3: Light curves from the beginning of accretion to $t=6 \times$ $10^{5} \mathrm{~s}$. The left upper mark of $\sharp a-1$ shows the light curve in Figure 4.

At the end, we obtain the isothermal temperature distribution of $\log T \sim 8.42$.

We repeatedly calculate normal bursts (combined hydrogen/helium burning) with use of the evolutionary code which includes the approximation reaction network. Though hydrogen consumption after a flash with use of this network might have been underestimated by $\sim 10 \%$ for $\log P \sim 22.8$ [14], remaining hydrogen is less than $\sim 1 \%$ in the bottom layer of normal bursts due to the convection. Therefore, in the deep region related to a superburst $(\log P>23)$, where rpprocess does not work anymore, our network can also be used except for detailed abundance distribution. Figure 3 shows the bursts from the beginning of accretion to $6 \times 10^{5} \mathrm{~s}$ with the accretion rate $5 \times 10^{-9} M_{\odot} \mathrm{yr}^{-1}$. In Figure 4 , we show the twelve bursts till $5.7 \times 10^{4} \mathrm{~s}$ which should be compared with those of Woosley et al. [15] having the solar initial composition and $1.75 \times 10^{-9} M_{\odot} \mathrm{yr}^{-1}$. In view of the fact that accretion rates are different from each other, both cases produce the regular bursts. In our case, these regular bursts continue $2.1 \times 10^{5} \mathrm{~s}$ and then the recurrence time becomes longer. Since there exists significant amount of produced materials inside the deep region, the heat transported to the inner part of the neutron star results in the lengthened recurrence intervals of bursts.

The totally calculated evolutionary time is $1.81 \times 10^{9} \mathrm{~s}$ and the total number of normal bursts amounts to 2786 . The time sequence of the bursts is illustrated in Figure 5. The time interval $\Delta t$ and the number of bursts are given in Table 1 for the individual period specified in terms of $\dot{M}$. Figures 68 show the light curve during the intervals (3-11), (11-15), and $(17.6-18.1) \times 10^{8}$ s. Critical bursts are marked by $\sharp 1-7$, whose profiles are discussed in Section 4.2. Important epochs leading to a superburst are specified as follows. The epoch $* \alpha$ is $930 \mathrm{~s}$ after the burst at $2 \times 10^{8} \mathrm{~s}$ during "period 1 ," and

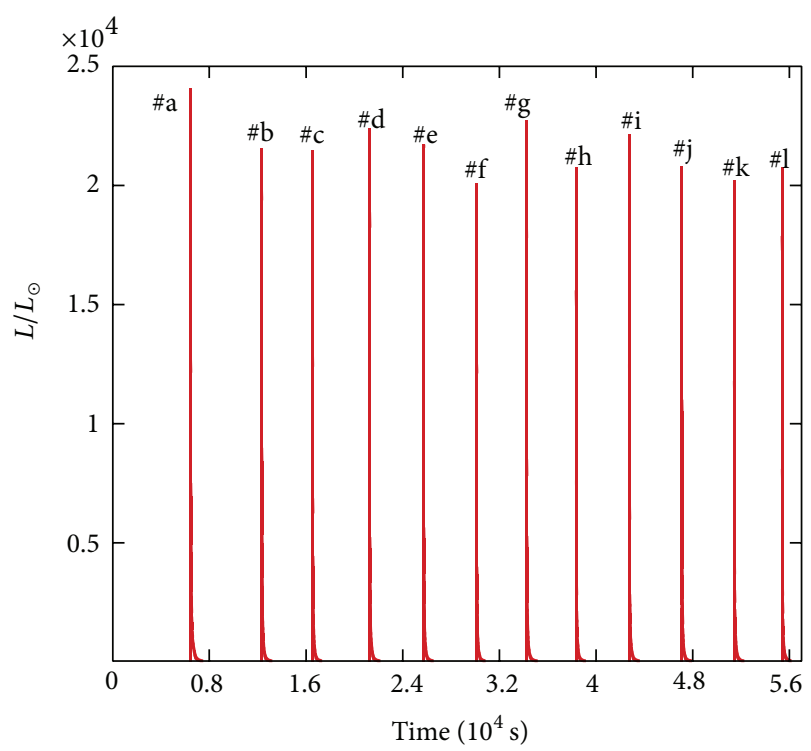

FIGURE 4: Light curves from the beginning to $t=5.7 \times 10^{4} \mathrm{~s}$. Marks of $\# a-1$ indicate each burst from the beginning of accretion $(t=0)$.

TABLE 1: Time catalogue corresponding to the accretion rates for three periods. $\Delta t$ is the time interval between the periods.

\begin{tabular}{lccc}
\hline Period & 1 & 2 & 3 \\
$\dot{M}\left(M_{\odot} \mathrm{yr}^{-1}\right)$ & $5 \times 10^{-9}$ & $1 \times 10^{-9}$ & $5 \times 10^{-9}$ \\
\hline Time $\left(10^{8} \mathrm{~s}\right)$ & $0-12$ & $12-16.8$ & $16.8-18.1$ \\
$\Delta t\left(10^{8} \mathrm{~s}\right)$ & 12 & 4.8 & 1.3 \\
Number of bursts & 1237 & 1416 & 133 \\
Specific stages of bursts & $* \alpha, \sharp 1-6$ & $* \beta$ & $\sharp 7, * \gamma, * \delta$ \\
\hline
\end{tabular}

the epoch $* \beta$ is $854 \mathrm{~s}$ after the burst at $1.5 \times 10^{9} \mathrm{~s}$ during "period 2." The epoch $* \gamma$ corresponds to the end of the last burst $\sharp 7$ and $* \delta$ is just before the ${ }^{12} \mathrm{C}+{ }^{12} \mathrm{C}$ ignition which is $5500 \mathrm{~s}$ after the epoch $* \gamma$.

Figure 9 shows temperature profiles against density and pressure, respectively, at the epochs $* \alpha-* \delta$. The ignition curve and deflagration line of the ${ }^{12} \mathrm{C}+{ }^{12} \mathrm{C}$ reaction are also shown in Figure 9. We note that in the context of detonations in superbursts, comparing $\tau_{12} \mathrm{C}+{ }^{12} \mathrm{C}$ to the dynamical time is discussed by Weinberg and Bildsten [62]. The two convexities near $\log \rho=6-7$ are due to the effect of the unstable hydrogen/helium burning. The energy generation rate is shown in Figure 10. Hot-CNO cycle, ${ }^{14} \mathrm{O}\left(\mathrm{e}^{+}, \nu\right){ }^{14} \mathrm{~N}(\mathrm{p}, \gamma)$ ${ }^{15} \mathrm{O}\left(\mathrm{e}^{+}, \nu\right){ }^{15} \mathrm{~N}(\mathrm{p}, \alpha){ }^{12} \mathrm{C}(\mathrm{p}, \gamma){ }^{13} \mathrm{~N}(\mathrm{p}, \gamma){ }^{14} \mathrm{O}$, has produced the energy of $10^{14} \mathrm{ergs}^{-1}$ for $\log P \leq 22$ [38]. Around $\log P=$ 27-28, the energy generation rates of $* \alpha, * \beta$, and $* \gamma$ before the ${ }^{12} \mathrm{C}+{ }^{12} \mathrm{C}$ ignition $* \delta$ become small because there remains small amount of fuel as the result of both the rp-process and steady burning of the ${ }^{12} \mathrm{C}+{ }^{12} \mathrm{C}$ reaction. It should be noted that the curve of $\varepsilon_{\mathrm{n}}$ for $* \delta$ falls down steeply around $\log P=26-26.5$ because of the significant decrease in abundances due to the sudden development of the convection as illustrated in Figure 14. 


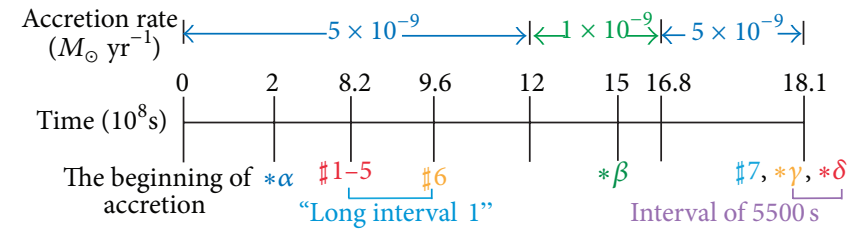

FIGURE 5: Time sequences of the models accompanied with normal bursts. When we start the accretion on the neutron star, time is set to be zero.

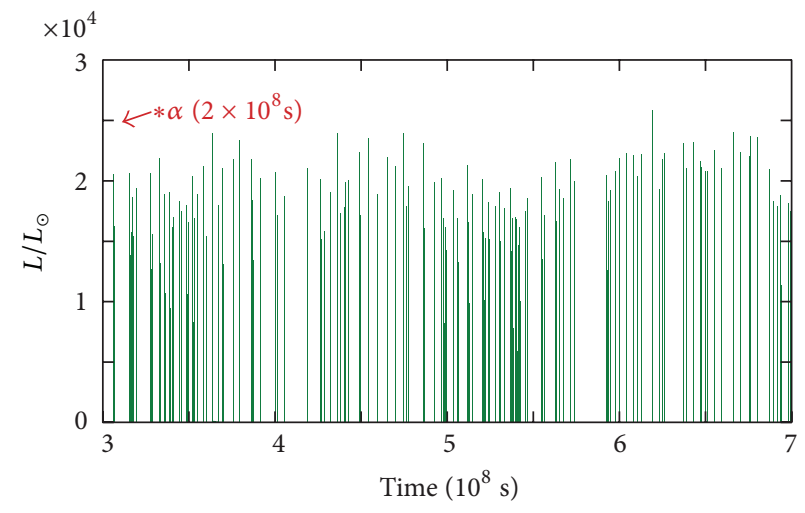

(a)

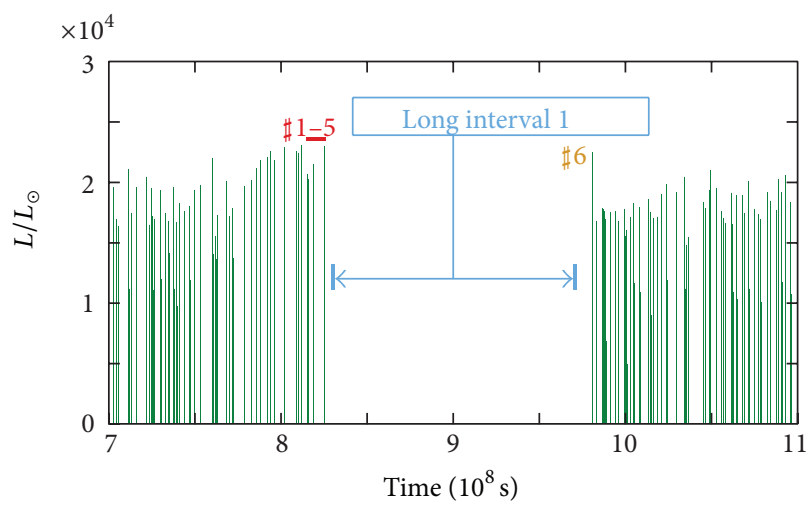

(b)

FIGURE 6: Light curves at $(3-7) \times 10^{8} \mathrm{~s}(\mathrm{a})$ and that from $(7-11) \times 10^{8} \mathrm{~s}(\mathrm{~b}) . * \alpha$ corresponds to the same epoch as shown by the mark in Figure 9. The interval between $(8.25-9.75) \times 10^{8} \mathrm{~s}$ is named the "log interval 1." Marks of $\sharp 1-5$ and $\sharp 6$ are successive numbers and a sign of each burst before and after "long interval 1," respectively.

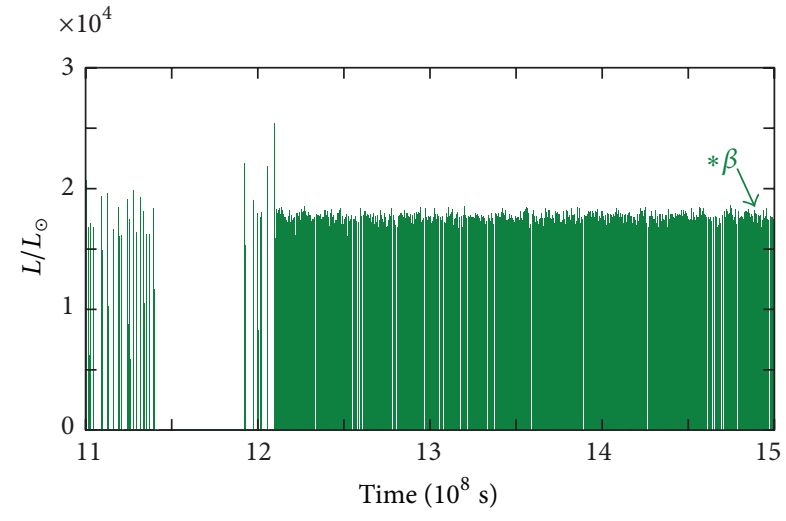

Figure 7: Light curves at $(11-15) \times 10^{8} \mathrm{~s} . * \beta$ indicates the same epoch as the mark in Figure 9.

For "period 1," the accretion rate is set to be $5 \times$ $10^{-9} M_{\odot} \mathrm{yr}^{-1}$ from the beginning of the accretion to $1.2 \times$ $10^{9} \mathrm{~s}$. The number of bursts occurred in this interval is 1237. The temperature distribution inside the deep accreted layers ( $\log \rho \geq 8$ and $\log P \geq 25$ ) remains isothermal of $\log T \sim 8.44$ (see the temperature distribution at $2 \times 10^{8} \mathrm{~s}$ in Figure 9). We can recognize that unstable combined hydrogen/helium burning has been generated for $\log \rho=6-6.5$ and $\log P=$ 22.5-23.5. Figure 11 shows the composition distribution at $2 \times 10^{8} \mathrm{~s}(* \alpha)$. The range of the pressure is equal to that in Figure 9. Around $\log P=22-23$, the rp-process produces

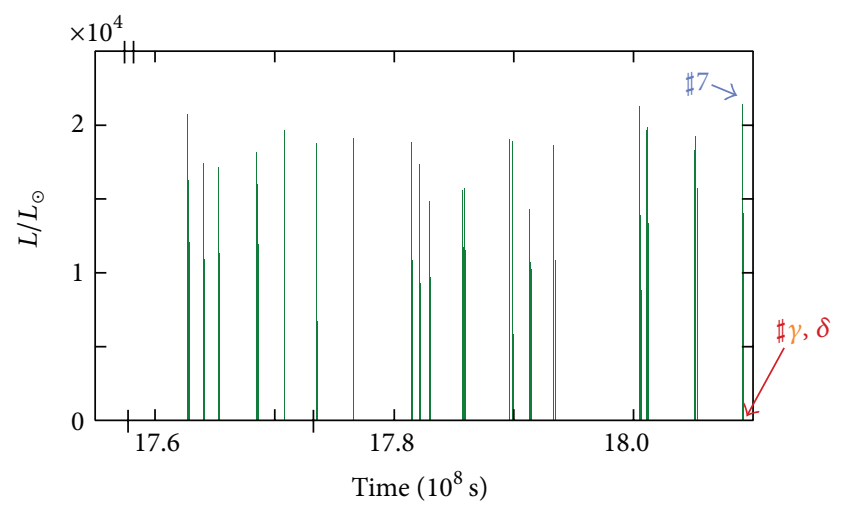

FIGURE 8: Light curves at $(17.6-18.1) \times 10^{8} \mathrm{~s} . \sharp 7$ indicates the last burst before the ignition of the carbon flash $(* \gamma$ and $* \delta)$.

both ${ }^{68} \mathrm{Ge}$ and ${ }^{64} \mathrm{Zn}$. Though there is no remained fuel of hydrogen [14], remained helium produces ${ }^{12} \mathrm{C}$ for $\log P>24$ (see the solid line in Figure 11) due to the steady burning. The increase in ${ }^{56} \mathrm{Ni}$ is ascribed to the numerical diffusion of the initial distribution and convective mixing at the beginning of the accretion for $\log P>26$; thus, we can consider that the mass fraction of ${ }^{56} \mathrm{Ni}$ in $26 \leq \log P \leq 27.5$ should be added to ${ }^{68} \mathrm{Ge}$. For "period 1," the carbon burning is stable $\left(\log P \sim 28, \log T \sim 8.4\right.$ and $\left.X\left({ }^{12} \mathrm{C}\right) \leq 0.01\right)$ in the sense that the increase in $T$ does not reach the ignition curve. In the bottom of the accretion layer ( $\log P \sim 28$ ), carbon 


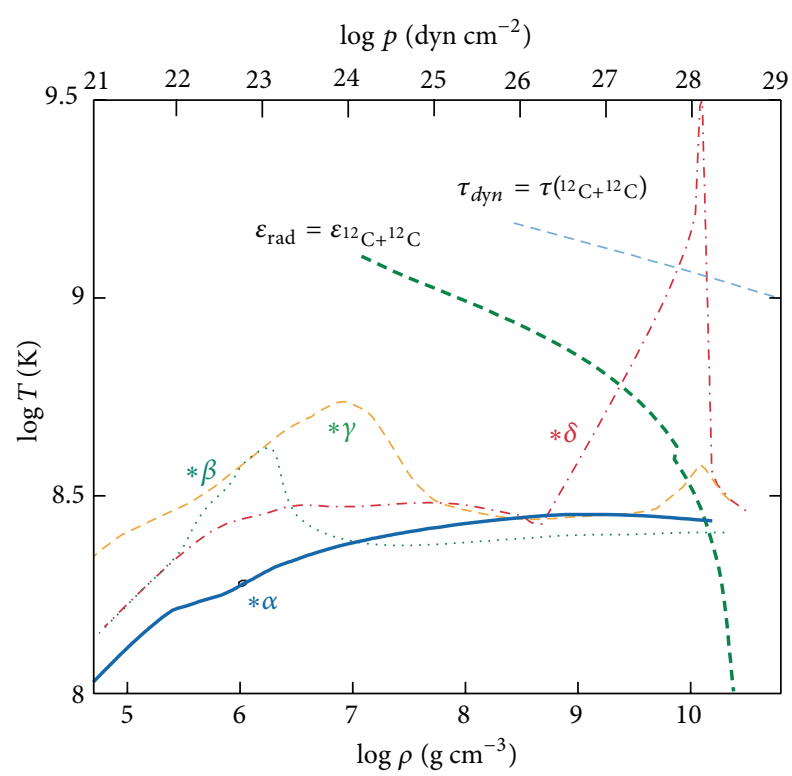

FIgURE 9: Temperature distribution against the density (bottom) and the pressure (top) at $2 \times 10^{8} \mathrm{~s}$ (solid line; $\left.* \alpha\right), 1.5 \times 10^{9} \mathrm{~s}$ (dotted line; $* \beta), 1.81 \times 10^{9} \mathrm{~s}$ (dashed line; $* \gamma$ ), and $5500 \mathrm{~s}$ from the $* \gamma$ (dotdashed line; $\delta$ ). At the stage $* \delta$, the ${ }^{12} \mathrm{C}+{ }^{12} \mathrm{C}$ reaction is ignited. Two lines on the right are ignition curves of ${ }^{12} \mathrm{C}+{ }^{12} \mathrm{C}$ reaction (dashed line, $\varepsilon_{\text {rad }}=\varepsilon^{12} \mathrm{C}+{ }^{12} \mathrm{C}$ ), and deflagration line (dashed line, $\tau_{\mathrm{dyn}}=$ $\left.\tau_{12} \mathrm{C}+{ }^{12} \mathrm{C}\right)$, with $X\left({ }^{12} \mathrm{C}\right)=0.1, X\left({ }^{56} \mathrm{Ni}\right)=0.9$.

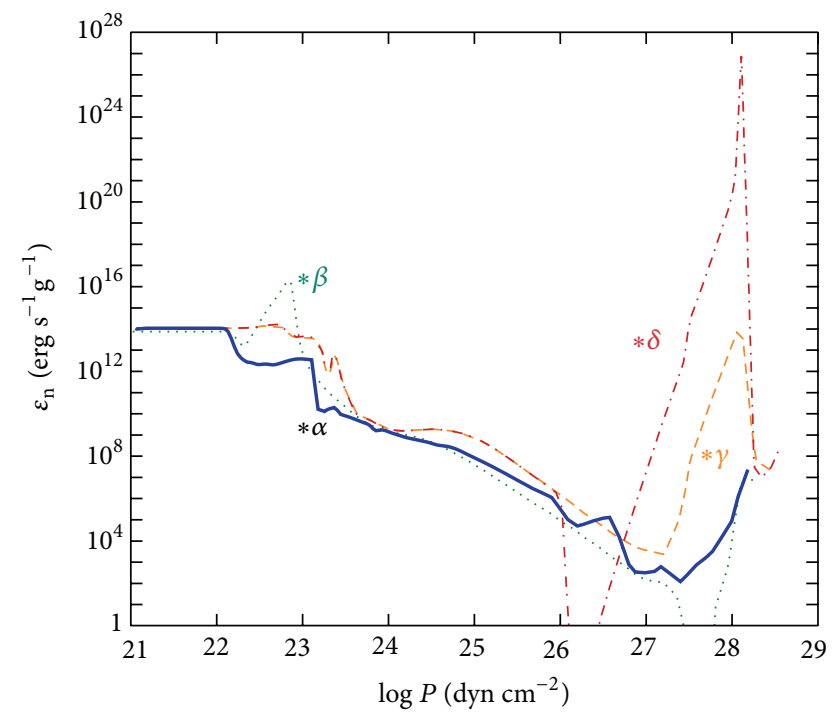

Figure 10: Nuclear energy generation rate versus pressure for four cases as shown in Figure 9.

sometimes burns intensively and the temperature of the layer has increased to $\log T \geq 8.6$. The amount of carbon is too small $\left(X\left({ }^{12} \mathrm{C}\right)<0.01\right)$ to trigger the nuclear flash. The nuclear energy generation for $* \alpha$ is supplied by helium burning for $\log P=23-25$, while it is supplied by reactions such as ${ }^{12} \mathrm{C}(\alpha, \gamma){ }^{16} \mathrm{O}$ and ${ }^{12} \mathrm{C}+{ }^{12} \mathrm{C}$ for $\log P=25-27.5$ (Figure 10). The crustal heating help $\varepsilon_{\mathrm{n}}$ to increase again for $\log P>27.5$.

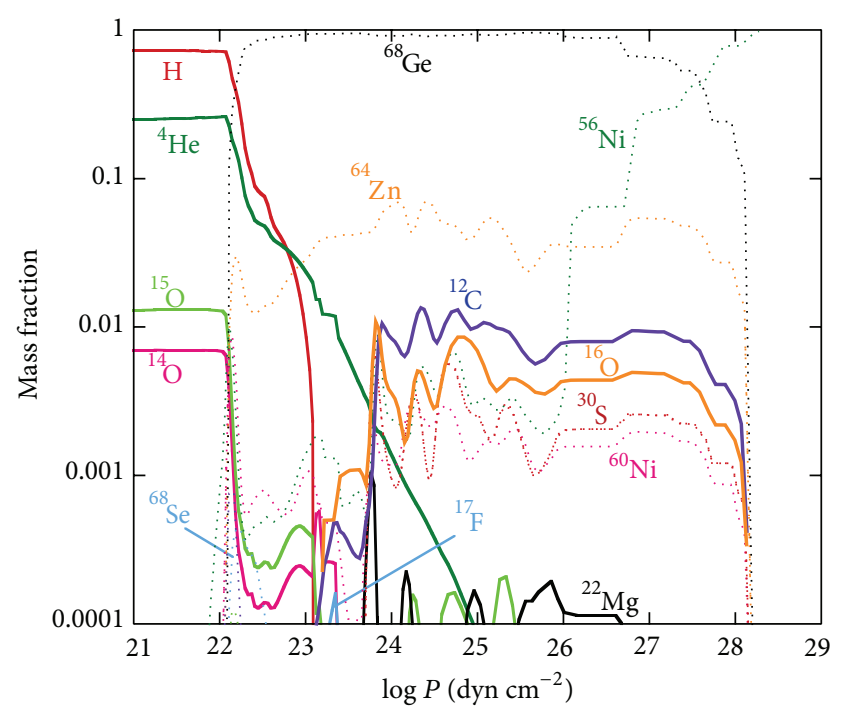

FIgure 11: Composition distribution at $2 \times 10^{8} \mathrm{~s}(* \alpha)$. For $\log P=$ $22-23$, the rp-process occurred and ${ }^{68} \mathrm{Ge}$ and ${ }^{64} \mathrm{Zn}$ are produced. For the relation between lines and composition, see Table 2 .

TABLE 2: Correspondence between lines and compositions.

\begin{tabular}{lcccccccc}
\hline Solid line & $\mathrm{H}$ & ${ }^{4} \mathrm{He}$ & ${ }^{12} \mathrm{C}$ & ${ }^{14} \mathrm{O}$ & ${ }^{15} \mathrm{O}$ & ${ }^{16} \mathrm{O}$ & ${ }^{17} \mathrm{~F}$ & ${ }^{22} \mathrm{Mg}$ \\
\hline Dotted line & ${ }^{30} \mathrm{~S}$ & ${ }^{56} \mathrm{Ni}$ & ${ }^{60} \mathrm{Zn}$ & ${ }^{60} \mathrm{Ni}$ & ${ }^{64} \mathrm{Ge}$ & ${ }^{64} \mathrm{Zn}$ & ${ }^{68} \mathrm{Se}$ & ${ }^{68} \mathrm{Ge}$ \\
\hline
\end{tabular}

After $t=1.2 \times 10^{9} \mathrm{~s}$, the accretion rate is artificially changed to $1 \times 10^{-9} M_{\odot} \mathrm{yr}^{-1}$. This results in the increase in ${ }^{12} \mathrm{C}$ abundance. We keep this accretion rate in the interval of $4.8 \times 10^{8} \mathrm{~s}$, and the 1416 bursts occur during this interval (period 2). The temperature distribution at $1.5 \times 10^{9} \mathrm{~s}$ for this accretion rate is shown by the dotted line in Figure 9 (denoted by $* \beta$ ). Figure 12 shows the composition distribution at this stage $(* \beta)$. For "period 2 ," hydrogen is consumed completely in the region of $\log P>23$ and the remained helium increases ${ }^{12} \mathrm{C}$ appreciably. The rp-process first produces ${ }^{68} \mathrm{GE}$ and ${ }^{64} \mathrm{Zn}$ and afterwards also ${ }^{60} \mathrm{Ni}$ and ${ }^{56} \mathrm{Ni}$. The appreciable decrease in $\varepsilon_{\mathrm{n}}$ corresponds to the dip of the composition distribution around $\log P \sim 27.4$ in Figure 12.

We have changed again the accretion rate to $\dot{M}=$ $5 \times 10^{-9} M_{\odot} \mathrm{yr}^{-1}$ and keep it for $1.3 \times 10^{8} \mathrm{~s}$ (period 3). The number of bursts is 133 in this interval. Though the temperature distribution has become lower compared with "period 1," if we continue the calculations of normal bursts, the temperature distribution should be recovered to that in "period 1." Therefore, we increase the crustal heating by a factor of 10 to save the computational time. Although this artificial change appears to be unreal, from the point of nuclear physics, physical process concerning the crustal heating has been rather uncertain; the rate is often multiplied some factors (see, e.g., $[8,63]$ ). We note that the heating rates (ergs/g) change up to 20 times for the pressures shown in tables of Haensel and Zdunik [57], and we have implemented the crustal heating using the tables as the heating source. 


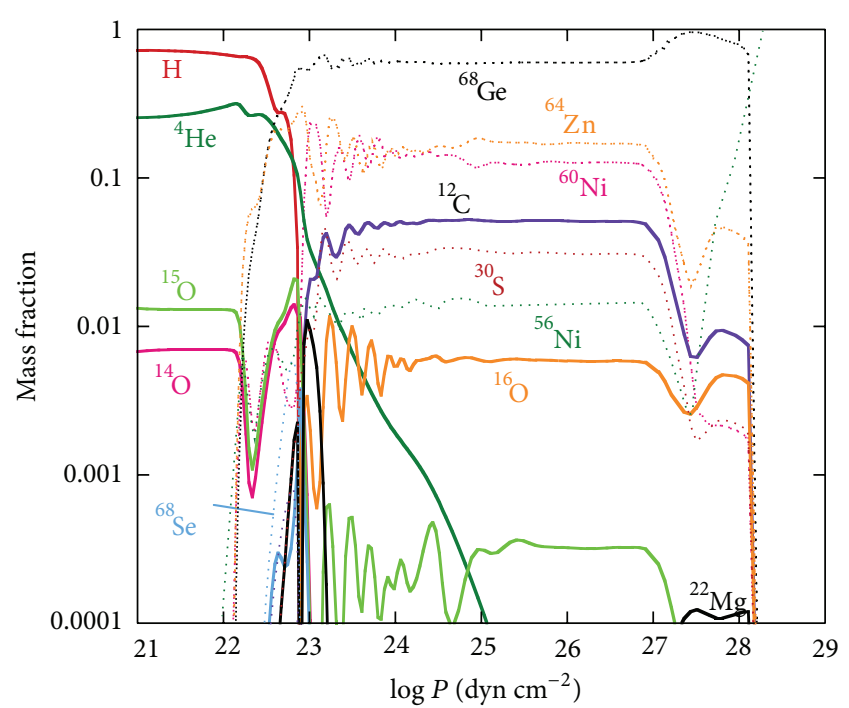

Figure 12: Composition distribution at $1.5 \times 10^{9} \mathrm{~s}(* \beta)$. The rpprocess forms ${ }^{68} \mathrm{Ge},{ }^{64} \mathrm{Zn}$ and ${ }^{60} \mathrm{Ni}$.

Figure 13 shows the composition distribution at the stage $(* \gamma)$. As in the case of "period 1 ," hydrogen is consumed in the bottom region of the hydrogen/helium burning and the remained helium forms ${ }^{12} \mathrm{C}$ continuously below the region. After $5500 \mathrm{~s}(* \delta)$ from the end $(* \gamma)$ of the last burst $(\sharp 7)$, we obtain the carbon flash. Finally, the ${ }^{12} \mathrm{C}+{ }^{12} \mathrm{C}$ reaction is successfully ignited in $\log P=28$ at the last epoch $(1.81 \times$ $\left.10^{9} \mathrm{~s}\right)$. The composition distributes uniformly for $\log P=$ 26-28 due to the convection as shown in Figure 14. We note that the ${ }^{12} \mathrm{C}+{ }^{12} \mathrm{C}$ flash does not yet develop to the peak of the total nuclear energy generation rate (Figure 19).

\subsection{Characteristic Features of Key Bursts Associated with Many} Normal Bursts. Let us discuss the profiles and/or sequences of the bursts for each period. In Figure 6(b), there is a significant interval at $8.25-9.75 \times 10^{8} \mathrm{~s}$. We call it the "long interval 1." We pay attention to five bursts ( $\sharp 1-5)$ before the "long interval 1" and the first burst ( $\$ 6$ ) after the interval.

Five bursts $\sharp 1-5$ extracted from Figure 6 are shown in Figure 15. "Long interval 1" begins at $8.252 \times 10^{8} \mathrm{~s}$. The light curve of the burst $\sharp 1$ is shown in Figures 16 and 17, where we set the time to be zero when the burst $\sharp 1$ starts. The roman numerals specify the epochs: I: just before the convection, II: peak of the total nuclear energy generation rate $L_{\mathrm{n} \text {,max }}$, and III: 1/10 of the maximum luminosity $L_{\max }$. In Figure 16, the epoch "IV" for the burst $\sharp 5$ corresponds to $220 \mathrm{~s}$ after the onset of the burst.

The shapes of the light curves and the products for the bursts $\sharp 1-4$ and $\sharp 6$ are almost same. The burst $\sharp 5$ is the longest burst compared with other bursts of $\sharp 1-4$ (Figure 16) and $\sharp 6$. At "IV" of the burst $\sharp 5$, the hydrogen burns in $\log P \leq 22.5$, and ${ }^{60} \mathrm{Zn}$ is produced where the convection occurs for $\log$ $P=22.2-23.2$ that leads to the exhaustion of hydrogen. The heat from this burning region of the convection is the reason for the long burst as seen in Figure 16, and the consumption

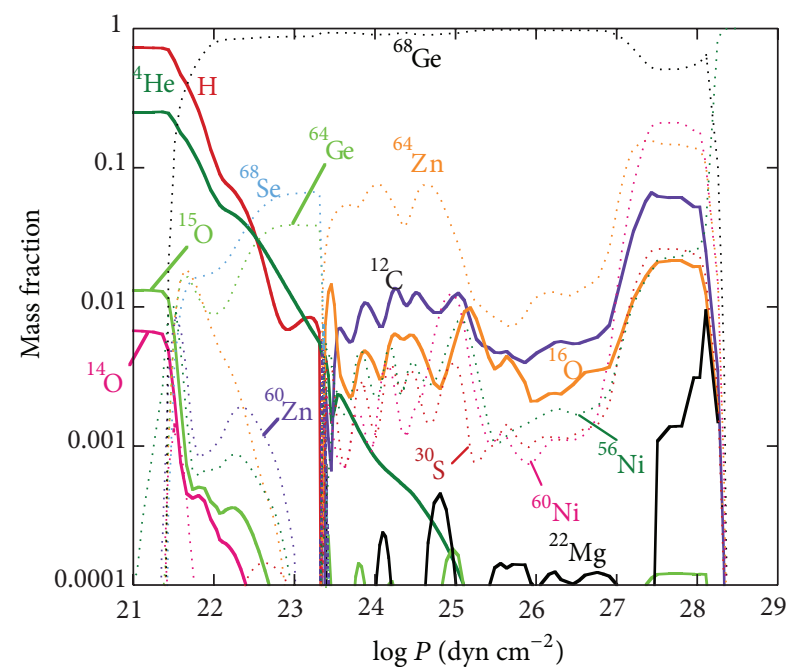

FIGURE 13: Composition distribution at $1.81 \times 10^{9} \mathrm{~s}(* \gamma)$. For $\log P=$ 21.6-23.3, the amount of ${ }^{68} \mathrm{Se}$ and ${ }^{64} \mathrm{Ge}$ becomes large temporarily due to the hydrogen/helium mixed burning.

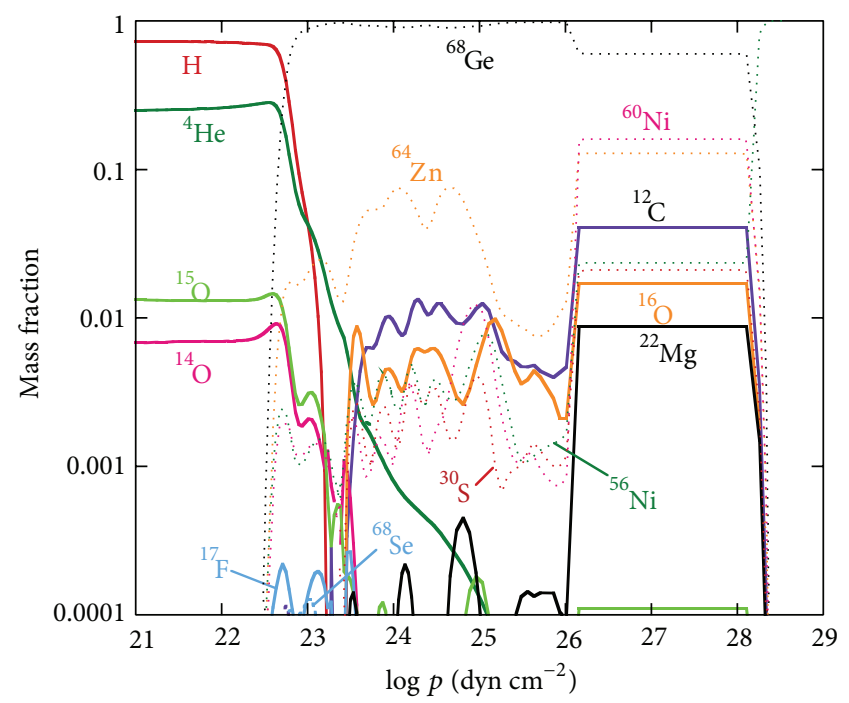

Figure 14: Composition distribution just after the ${ }^{12} \mathrm{C}+{ }^{12} \mathrm{C}$ ignition $(* \delta)$.

of fuels leads to the long interval after $\sharp 5$ (Figures 6(b) and 15). This feature can be seen in other sequences of bursts. At the epoch "IV" of the burst $\sharp 3$ (2240 s after the onset of the burst), the convection also occurs between $\log P=22.5-23$ where hydrogen is completely consumed. Therefore, as seen in Figure 15 the interval to the next burst $\sharp 4$ becomes long compared to other intervals except for the "long interval 1 ," because the accumulated hydrogen has been consumed. The same situation also occurs for the rather long interval $1.14-1.19 \times 10^{9} \mathrm{~s}$ in Figure 7 .

The luminosity (Figure 17) in the burst $* \beta$ of $\dot{M}=1 \times$ $10^{-9} M_{\odot} \mathrm{yr}^{-1}$ is a little small compared with the bursts $\sharp 1-5$ and $\sharp 6$ of $\dot{M}=5 \times 10^{-9} M_{\odot} \mathrm{yr}^{-1}$. Though the products of ${ }^{68} \mathrm{Ge},{ }^{68} \mathrm{Se},{ }^{64} \mathrm{Zn}$, and ${ }^{22} \mathrm{Mg}$ are the same as those in 


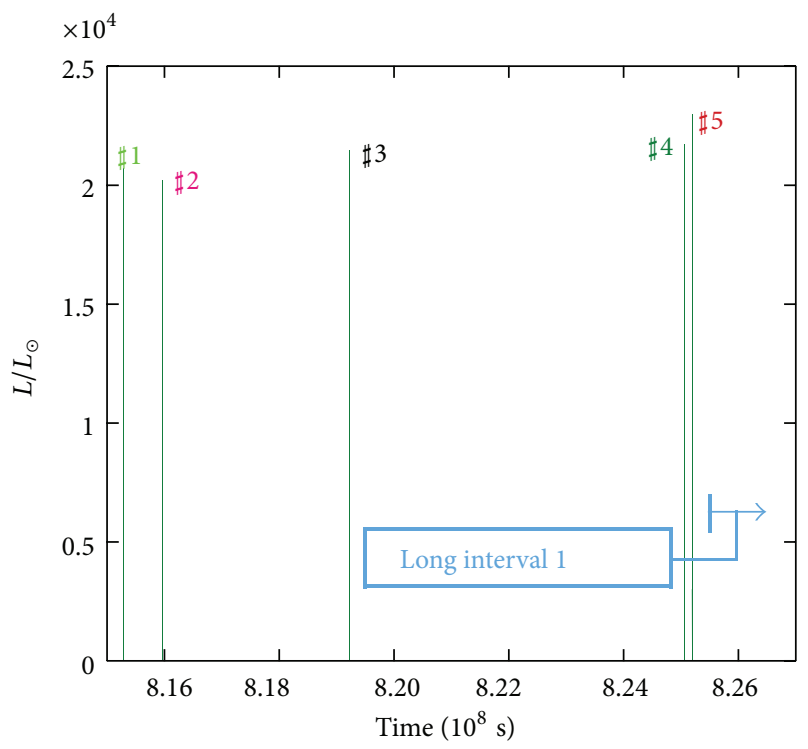

FIGURE 15: Light curves at $8.15-8.27 \times 10^{8}$ s. Each number from $\sharp 1-5$ corresponds to each burst. "Long interval 1" begins after the elapsed time $8.252 \times 10^{8} \mathrm{~s}(\sharp 5)$. Detailed profiles of the burst $\sharp 1$ and 5 are shown in Figure 16.

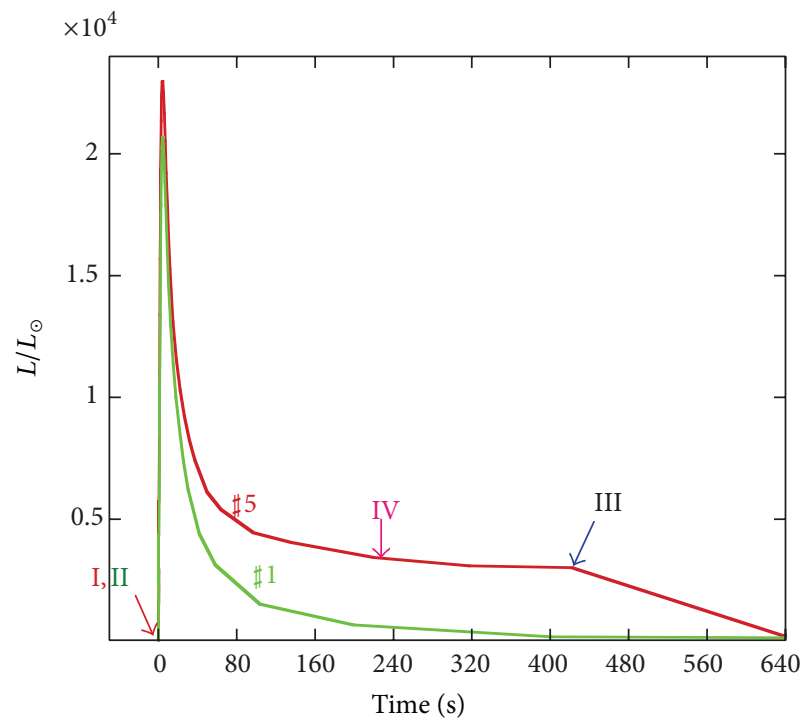

FIGURE 16: Light curves of the burst $\sharp 1$ and burst $\sharp 5$ that correspond to Figure 15. IV is the time of $220 \mathrm{~s}$ after the onset of the burst. The burst $\sharp 5$ is the longest burst compared with other bursts $\sharp 1-4$. For comparison, the burst $\sharp 1$ is also shown.

the other bursts, the amount of heavy elements like ${ }^{60} \mathrm{Ni}$ and ${ }^{64} \mathrm{Zn}$ are produced more than those of the other bursts. The temperature distribution of the inner accretion layer $(\log P=24-28)$ is lower than that of the other bursts with $\dot{M}=5 \times 10^{-9} M_{\odot} \mathrm{yr}^{-1}$ (see Figure 9, where $T$ in the stage of $* \beta$ for $\log P>24$ is low due to the conduction). We can consider that due to the increased conduction the luminosity in the bursts decreases because the heat from the combined hydrogen/helium burning has flowed into the

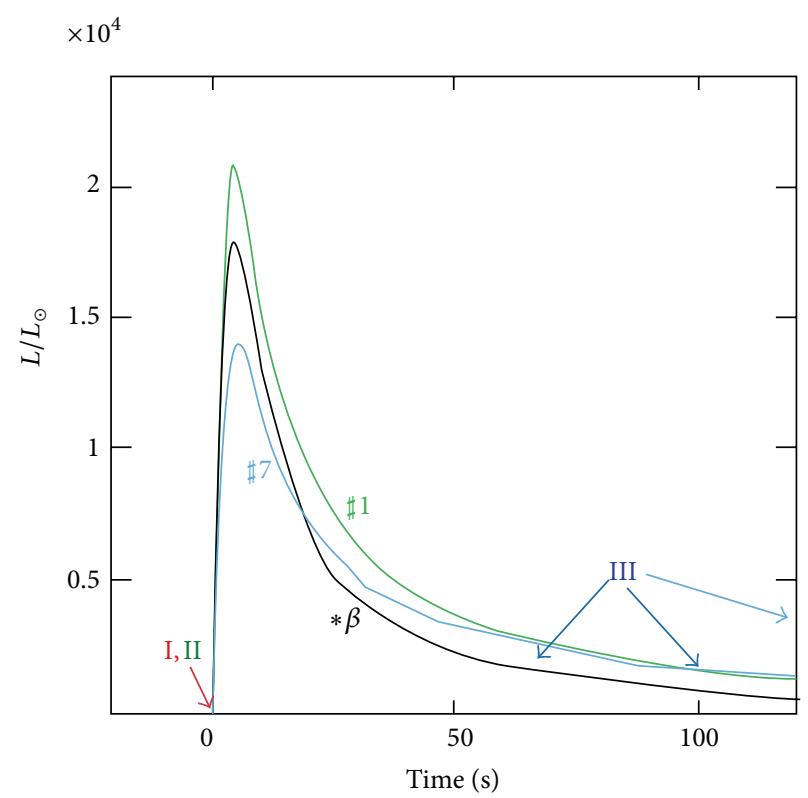

FIGURE 17: Light curves of the bursts $\sharp 1, * \beta$, and $\sharp 7$. Roman numerals are corresponded with the time of I: just before the start of convection, II: $L_{\mathrm{n}, \max }$, and III: $1 / 10$ of $L_{\max }$. The epoch III in $\sharp 7$ is $154 \mathrm{~s}$ after the onset of the burst.

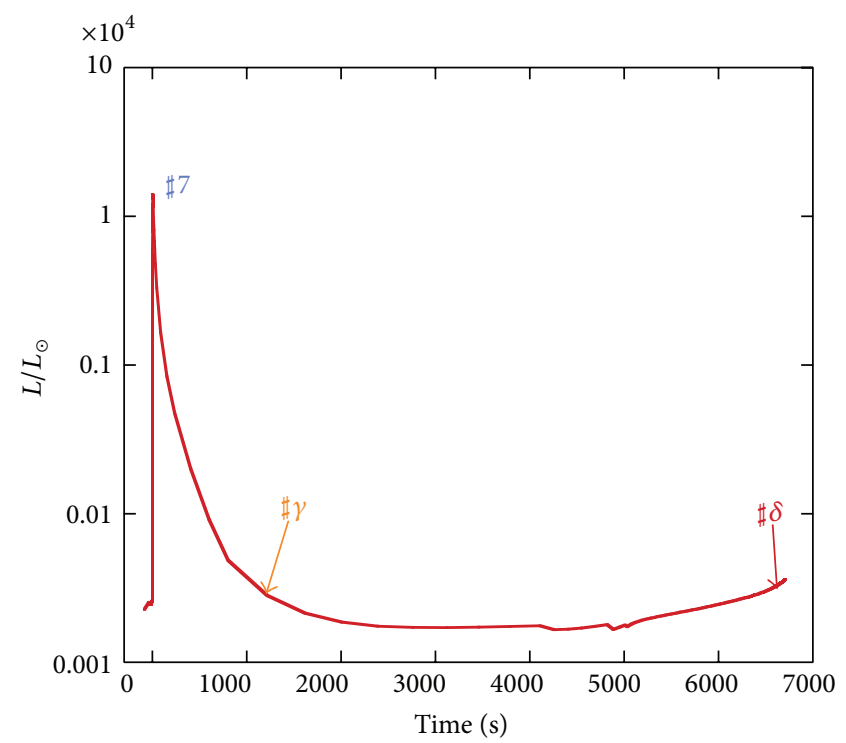

FIGURE 18: Light curve of the burst $\sharp 7$ and the onset of the carbon flash. $\sharp \gamma$ and $\sharp \delta$ correspond to the same epoch as shown by the mark in Figure 9.

core. This phenomena can be understood from the idea of watershed introduced by Fujimoto et al. [35]. As illustrated in Figure 17, the last burst $\sharp 7$ before the carbon ignition is weaker in strength than that of the burst $* \beta$, because the hydrogen/helium burning is ignited at the deeper region (log $P=23.3$ ), which can be seen from the ash of the rp-process ${ }^{68} \mathrm{Se}$ in Figure 13, compared to the other bursts $(\log P=22.9)$ as seen in Figures 11 and 12. 


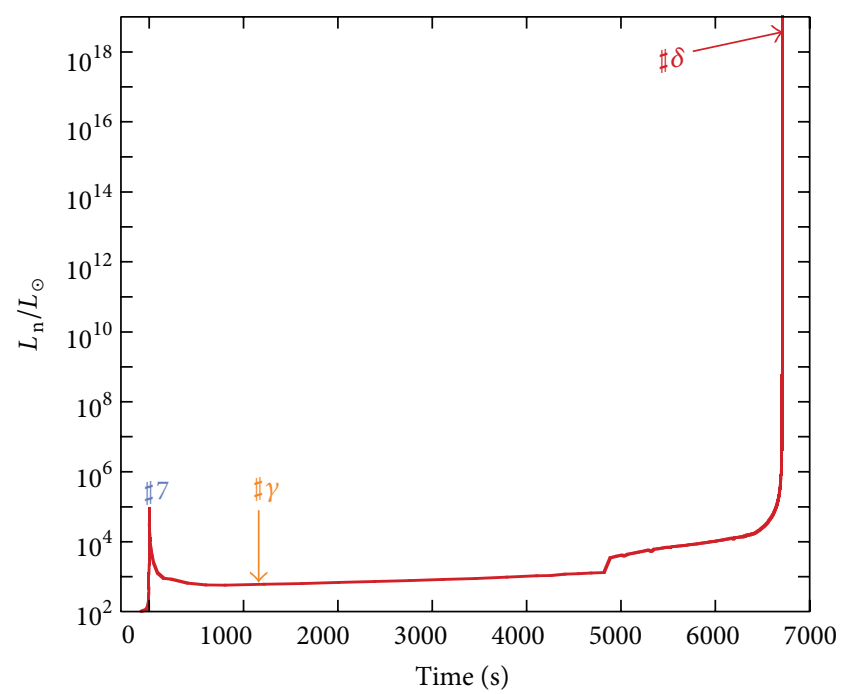

Figure 19: Total nuclear energy generation rate for the burst $\sharp 7$ and the onset of the carbon flash.

The light curve and the total nuclear energy generation rate, $L_{n}$, from the burst $\sharp 7$ to the onset of the carbon flash are shown in Figures 18 and 19. Just after the burst, $* \gamma$, the convection occurs at the narrow region of $\log P=27.9-28$ (Figure 13). At $\simeq 5300 \mathrm{~s}$ in Figure 19, the convection begins to spread to lower pressure region, and it extends from log $P=28$ to 26 as shown in Figure 14. We note that $L_{\mathrm{n}}$ at $* \delta$ is not attained to the $L_{\mathrm{n} \text {,max }}$. However, since the temperature in $\log P \geq 27.8$ has increased beyond the deflagration temperature defined by $\tau_{\mathrm{dyn}}=\tau_{12} \mathrm{C}+^{12} \mathrm{C}$ as seen from Figure 9 , we can infer that the flash should develop to a dynamical phenomena of deflagration. Finally, we stop the calculation of the flash due to the numerical difficulty in the assumption of the hydrostatical equilibrium; nevertheless, we insist that this flash could become a superburst.

\section{Concluding Remarks}

We have presented the two cases for the models of superbursts: single helium flash and carbon flash accompanied with many normal bursts. For the helium flash, the burst has a long duration time although the accretion rate is different from the observation. We suggest that the helium flash could originate the superburst if the burning develops to the deflagration and/or detonation. For a single carbon flash, the temperature does not reach the deflagration temperature due to the limit of heat conduction.

For the carbon flash accompanied with many normal bursts, we carried out the successive 2786 normal bursts up to the time of $1.81 \times 10^{9} \mathrm{~s}$ using the observed accretion rates. We showed the profiles of the several normal bursts and the onset of the carbon flash after the normal bursts. Since there exists significant amount of produced elements in the deeper than the region where hydrogen/helium burning occurred, the heat transported to the core by them lengthens the recurrence intervals of bursts. We have also shown that the recurrence time becomes longer than other bursts when the little burning occurred for the bottom of the hydrogen/helium burning layer after the main burning had ceased. This leads to the consumption of nuclear fuels of $\mathrm{H}$ and $\mathrm{He}$ and increases the products of rp-process. We have recognized that "long interval 1" is a remarkable case for the delay to the next burst due to the above reasons. Before the carbon flash, we find various profiles of the light curve and different intervals between bursts; Quantitative comparison with the observations should shed a new insight into the X-ray burst research. Since normal bursts have been observed before the superbursts, our scenario is consistent with the observations. We conclude that a carbon flash accompanied with many normal bursts should trigger a superburst. It is interesting to apply the above idea to a superburst of a helium accretor like 4U1820-30.

For the helium flash shown in Section 3, accretion rate to produce a superburst is rather low compared with observations. If we repeat normal helium flashes with the observed $\dot{M}$ as demonstrated in Section 4.2, a superburst could be triggered in a deep region of the accreting layers. Finally, we remark that to get a detailed history toward the superburst, it is needed to calculate many bursts using the code of the neutron star evolution with a large nuclear reaction network coupled.

\section{Conflict of Interests}

The authors declare that there is no conflict of interests regarding the publication of this paper.

\section{References}

[1] H. Schatz, L. Bildsten, A. Cumming, and M. Ouellette, "Nuclear physics in normal X-ray bursts and superblasts," Nuclear Physics A, vol. 718, pp. 247-254, 2003.

[2] A. Cumming, J. Macbeth, J. J. M. in't Zand, and D. Page, "Long type I x-ray bursts and neutron star interior physics," The Astrophysical Journal, vol. 646, pp. 429-451, 2006.

[3] A. Parikh, J. José, G. Sala, and C. Iliadis, "Nucleosynthesis in type I X-ray bursts," Progress in Particle and Nuclear Physics, vol. 69, no. 1, pp. 225-253, 2013.

[4] L. Keek, A. Heger, and J. J. M. in't Zand, "Superburst models for neutron stars with hydrogen- and helium-rich atmospheres," The Astrophysical Journal, vol. 752, no. 2, 150, 2012.

[5] R. Wijnands, "Recurrent very long type I X-ray bursts in the low-mass X-ray binary 4U 1636-53," The Astrophysical Journal Letters, vol. 554, no. 1, article L59, 2001.

[6] T. E. Strohmayer and C. B. Markwardt, "Evidence for a millisecond pulsar in $4 \mathrm{U}$ 1636-53 during a superburst," Astrophysical Journal Letters, vol. 577, no. 1, pp. 337-345, 2002.

[7] A. Cumming and J. Macbeth, "The thermal evolution following a superburst on an accreting neutron star," Astrophysical Journal Letters, vol. 603, no. 1, pp. L37-L40, 2004.

[8] L. Keek and A. Heger, "Multi-zone models of superbursts from accreting neutron stars," Astrophysical Journal, vol. 743, no. 2, article 189, 2011.

[9] T. E. Strohmayer and E. F. Brown, "A remarkable 3 hour thermonuclear burst from 4U 1820-30," The Astrophysical Journal, vol. 566, pp. 1045-1059, 2002. 
[10] E. Kuulkers, "A superburst from GX 3+1," Astronomy and Astrophysics, vol. 383, no. 1, pp. L5-L8, 2002.

[11] L. Keek, "Photospheric radius expansion in superburst precursors from neutron stars," The Astrophysical Journal, vol. 756, article 130, 2012.

[12] A. Cumming and L. Bildsten, "Carbon flashes in the heavyelement ocean on accreting neutron stars," Astrophysical Journal Letters, vol. 559, no. 2, pp. L127-L130, 2001.

[13] A. Cumming, "Models of type I X-RAY bursts from $4 \mathrm{U} 1820$ 30," The Astrophysical Journal, vol. 595, no. 2, pp. 1077-1085, 2003.

[14] O. Koike, M. Hashimoto, R. Kuromizu, and S. Fujimoto, "Final products of the rp-process on accreting neutron stars," The Astrophysical Journal, vol. 603, no. 1, pp. 242-251, 2004.

[15] S. E. Woosley, A. Heger, and A. Cumming, "Models for type I X-ray bursts with improved nuclear physics," The Astrophysical Journal Supplement Series, vol. 151, no. 1, p. 75, 2004.

[16] R. Cornelisse, E. Kuulkers, J. J. M. in’t Zand, F. Verbunt, and J. Heise, "A four-hours long burst from Serpens X-1," Astronomy \& Astrophysics, vol. 382, pp. 174-177, 2002.

[17] E. Kuulkers, J. J. M. In't Zand, and M. H. van Kerkwijk, "A half-a-day long thermonuclear X-ray burst from KS 1731-260," Astronomy and Astrophysics, vol. 382, no. 2, pp. 503-512, 2002.

[18] J. J. M. In't Zand, E. Kuulkers, F. Verbunt, J. Heise, and R. Cornelisse, "A superburst from 4U 1254-69," Astronomy and Astrophysics, vol. 411, no. 3, pp. L487-L491, 2003.

[19] J. J. M. in't Zand, R. Cornelisse, and A. Cumming, "Superbursts at near-Eddington mass accretion rates," Astronomy \& Astrophysics, vol. 426, no. 1, pp. 257-265, 2004.

[20] H. Schatz, L. Bildsten, A. Cumming, and M. Wiescher, "The rapid proton process ashes from stable nuclear burning on an accreting neutron star," Astrophysical Journal Letters, vol. 524, no. 2, pp. 1014-1029, 1999.

[21] H. Worpel, D. K. Galloway, and D. J. Price, "Evidence for accretion rate change during type i X-ray bursts," Astrophysical Journal, vol. 772, no. 2, article 94, 2013.

[22] A. Bahramian, C. O. Heinke, and G. R. Sivakoff, "Discovery of the third transient X-ray binary in the galactic globular cluster Terzan 5," The Astrophysical Journal, vol. 780, no. 2, article 127, 2014.

[23] H. Schatz, L. Bildsten, and A. Cumming, "Photodisintegrationtriggered nuclear energy release in superbursts," Astrophysical Journal Letters, vol. 583, no. 2, pp. L87-L90, 2003.

[24] M. Sinha, M. Dey, S. Ray, and J. Dey, "Super bursts and long bursts as surface phenomena of compact objects," Monthly Notices of the Royal Astronomical Society, vol. 337, no. 4, pp. 1368-1372, 2002.

[25] C. M. Malone, A. Nonaka, A. S. Almgren, J. B. Bell, and M. Zingale, "Multidimensional modeling of type I X-ray bursts. I. Two-dimensional convection prior to the outburst of a pure ${ }^{4} \mathrm{He}$ accretor," The Astrophysical Journal, vol. 728, no. 2, article 118, 2011.

[26] C. M. Malone, M. Zingale, A. Nonaka, A. S. Almgren, and J. B. Bell, "Multidimensional modeling of type I X-ray bursts. II. Two-dimensional convection in a mixed $\mathrm{H} / \mathrm{He}$ accretor," The Astrophysical Journal, vol. 788, article 115, 2014.

[27] Y. Cavecchi, A. L. Watts, J. Braithwaite, and Y. Levin, "Flame propagation on the surfaces of rapidly rotating neutron stars during type I X-ray bursts," submitted, http://arxiv.org/abs/1212 .2872 .
[28] R. V. E. Lovelace, A. K. Kulkarni, and M. M. Romanova, "Torsional magnetic oscillations in type I X-ray bursts," The Astrophysical Journal, vol. 656, no. 1, pp. 393-398, 2007.

[29] D. J. B. Payne and A. Melatos, "Magnetic burial and the harmonic content of millisecond oscillations in thermonuclear X-ray bursts," The Astrophysical Journal, vol. 652, no. 1, p. 597, 2014.

[30] R. Artigue, D. Barret, F. K. Lamb, K. H. Lo, and M. C. Miller, "Testing the rotating hotspot model using X-ray burst oscillations from 4U 1636-536," Monthly Notices of the Royal Astronomical Society, vol. 433, no. 1, pp. L64-L68, 2013.

[31] M. Fujimoto, T. Hanawa, I. Iben Jr., and M. B. Richardson, "Thermal evolution of accreting neutron stars. II-long X-ray bursts as a probe into the interior," The Astrophysical Journal, vol. 315, pp. 198-208, 1987.

[32] K. S. Thorne, "The relativistic equations of stellar structure and evolution," Astrophysical Journal, vol. 212, pp. 825-831, 1977.

[33] D. Sugimoto, K. Nomoto, and Y. Eriguchi, "Stable numerical method in computation of stellar evolution," Progress of Theoretical Physics Supplement, vol. 70, pp. 115-131, 1981.

[34] M. Y. Fujimoto and D. Sugimoto, "Helium shell flashes and evolution of accreting white dwarfs," The Astrophysical Journal, vol. 257, pp. 291-302, 1982.

[35] M. Fujimoto, T. Hanawa, I. Iben Jr., and M. B. Richardson, "Thermal evolution of accreting neutron stars," The Astrophysical Journal, vol. 278, pp. 813-824, 1984.

[36] T. Hanawa and M. Y. Fujimoto, "Thermal response of neutron stars to shell flashes," Publication of the Astronomical Society of Japan, vol. 36, pp. 199-214, 1984.

[37] C. Angulo, M. Arnould, and M. Rayet, "A compilation of charged-particle induced thermonuclear reaction rates," Nuclear Physics A, vol. 656, no. 1, pp. 3-183, 1999.

[38] R. K. Wallace and S. E. Woosley, "Explosive hydrogen burning," Astrophysical Journal, vol. 45, p. 389, 1981.

[39] T. Hanawa, D. Sugimoto, and M. A. Hashimoto, "Nucleosynthesis in explosive hydrogen burning and its implications in ten-minute interval of X-ray bursts," Publications of the Astronomical Society of Japan, vol. 35, pp. 491-506, 1983.

[40] W. L. Slattery, G. D. Doolen, and H. E. Dewitt, "Improved equation of state for the classical one-component plasma," Physical Review A, vol. 21, no. 6, pp. 2087-2095, 1980.

[41] M. B. Richardson, H. M. Van Horn, K. F. Ratcliff, and R. C. Malone, "Neutron star evolutionary sequences," The Astrophysical Journal, vol. 255, pp. 624-653, 1982.

[42] V. Canuto, "Equation of state at ultrahigh sensities," Annual Review of Astronomy and Astrophysics, vol. 12, pp. 167-214, 1974.

[43] V. Canuto, "Equation of state at ultrahigh densities," Annual Review of Astronomy and Astrophysics, vol. 13, pp. 335-380, 1975.

[44] B. L. Friman and O. V. Maxwell, "Neutrino emissivities of neutron stars," The Astrophysical Journal, vol. 232, pp. 541-557, 1979.

[45] G. G. Festa and M. A. Ruderman, "Neutrino-pair bremsstrahlung from a degenerate electron gas," Physical Review, vol. 180, no. 5, pp. 1227-1231, 1969.

[46] G. Beaudet, V. Petrosian, and E. E. Salpeter, "Energy losses due to neutrino processes," The Astrophysical Journal, vol. 150, p. 979, 1967.

[47] R. C. Malone, Cooling of superfluid neutron stars [Ph.D thesis], Cornell University, Ithaca, NY, USA, 1974.

[48] G. Baym, C. Pethick, and D. Pines, "Electrical conductivity of neutron star matter," Nature, vol. 224, pp. 674-675, 1969. 
[49] E. Flowers and N. Itho, "Transport properties of dense matter," The Astrophysical Journal, vol. 206, pp. 218-242, 1976.

[50] N. Itoh, N. Matsumoto, M. Seki, and Y. Kohyama, "Neutrinopair bremsstrahlung in dense stars. II-crystalline lattice case," The Astrophysical Journal, vol. 279, pp. 413-418, 1984.

[51] I. Iben Jr., "Thermal pulses; p-capture, alpha-capture, s-process nucleosynthesis; and convective mixing in a star of intermediate mass," The Astrophysical Journal, vol. 196, part 1, pp. 525-547, 1975.

[52] A. N. Cox and J. N. Stewart, "Rosseland opacity tables for population I compositions," The Astrophysical Journal Supplement, vol. 19, p. 243, 1970.

[53] A. N. Cox and J. N. Stewart, "Rosseland opacity tables for population II compositions," The Astrophysical Journal, vol. 19, Supplement, p. 261, 1970.

[54] W. B. Hubbard and M. Lampe, "Thermal conduction by electrons in stellar matter," The Astrophysical Journal Supplement Series, vol. 18, p. 297, 1969.

[55] V. Canuto, "Electrical conductivity and conductive opacity of a relativistic electron gas," The Astrophysical Journal, vol. 159, pp. 641-652, 1970.

[56] H. E. Dewitt, H. C. Gravoske, and M. S. Cooper, "Screening factors for nuclear reactions. I. General theory," The Astrophysical Journal, vol. 181, pp. 439-456, 1973.

[57] P. Haensel and J. L. Zdunik, "Non-equilibrium processes in the crust of an accreting neutron star," Astronomy and Astrophysics, vol. 227, no. 2, pp. 431-436, 1990.

[58] D. K. Galloway, M. P. Muno, J. M. Hartman, D. Psaltis, and D. Chakrabarty, "Thermonuclear (type i) X-ray bursts observed by the rossi X-ray timing explorer," Astrophysical Journal, vol. 179, no. 2, pp. 360-422, 2008.

[59] E. Kuulkers, J. J. M. in't Zand, and J.-L. Atteia, "What ignites on the neutron star of 4U 0614+091?" Astronomy \& Astrophysics, vol. 514, article A65, 2010.

[60] L. Bildsten, Thermonuclear Burning on Rapidly Accreting Neutron Stars, Kluwer Academic Publishers, Dordrecht, The Netherlands, 1998.

[61] E. P. J. van den Heuvel, in Proceedings of the 2nd BeppoSAX Meeting, E. P. J. van den Heuvel, J. J. M. In't Zand, and R. A. M. J. Wijers, Eds., 2003.

[62] N. N. Weinberg and L. Bildsten, "Carbon detonation and shock-triggered helium burning in neutron star superbursts," Astrophysical Journal Letters, vol. 670, no. 2, pp. 1291-1300, 2007.

[63] Y. Matsuo, H. Tsujimoto, and T. Noda, "Effects of a new triple$\alpha$ reaction on $\mathrm{x}$-ray bursts of a helium-accreting neutron star," Progress of Theoretical Physics, vol. 126, article 1177, 2011. 

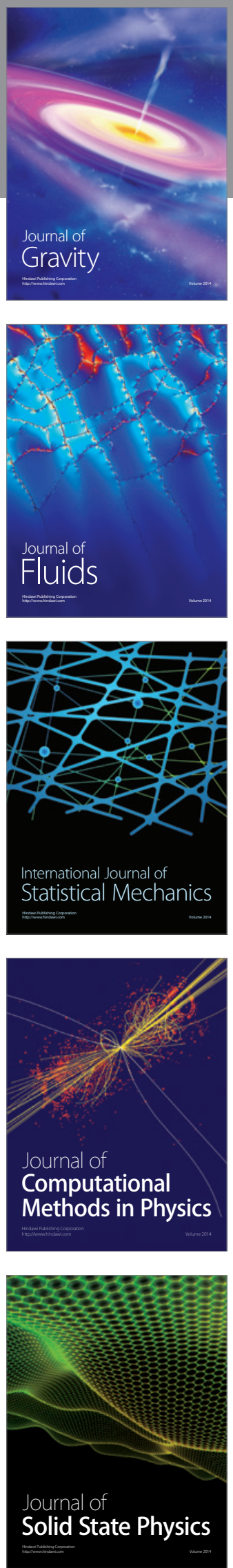

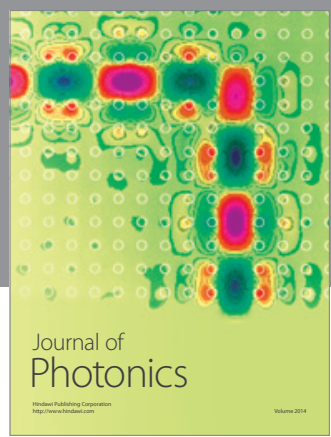

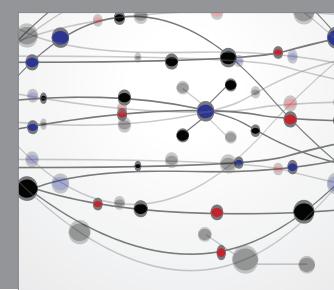

The Scientific World Journal

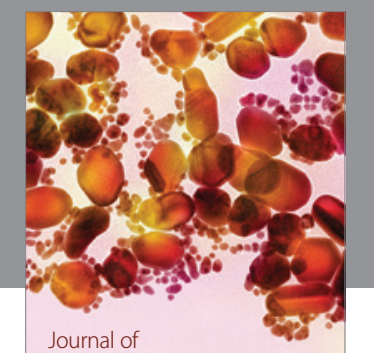

Soft Matter
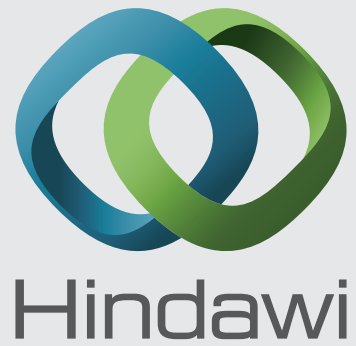

Submit your manuscripts at

http://www.hindawi.com
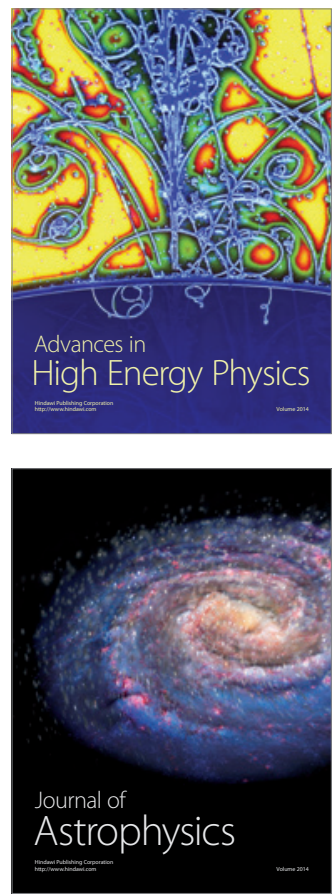
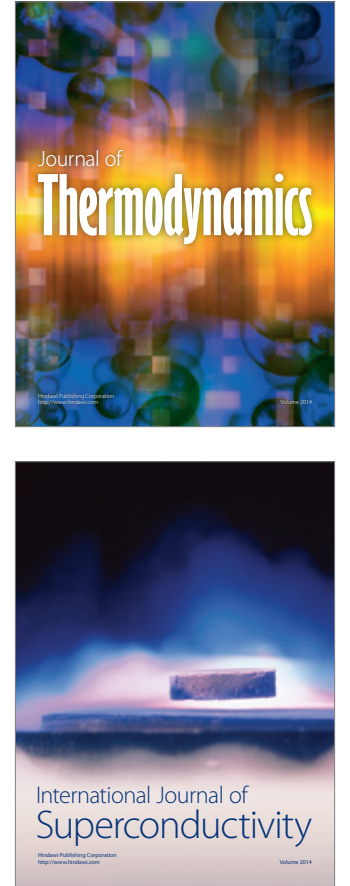
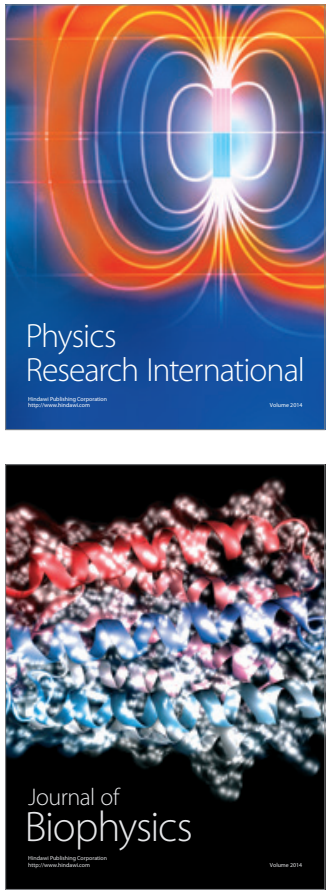
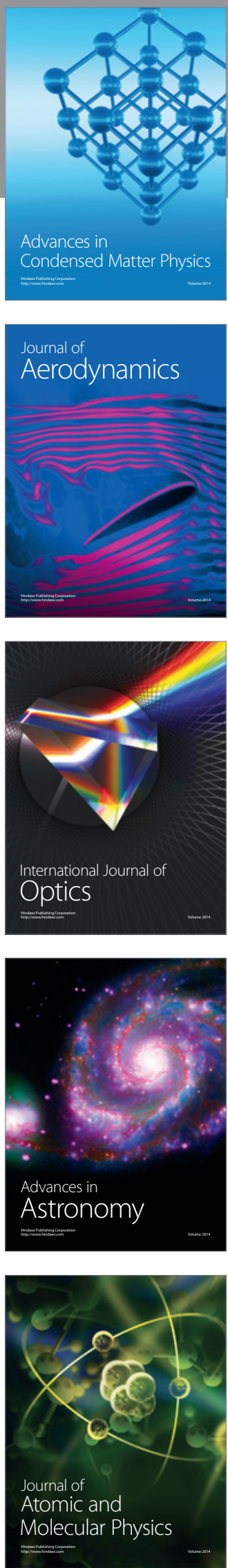